\title{
HOW SEDLÁČEK'S BLADELESS TURBINE WORKS?
}

\author{
Ondřej Kincl ${ }^{1}$, Michal Pavelka ${ }^{1}$, František Maršík ${ }^{2}$, Miroslav Sedláček ${ }^{3}$ \\ 1 Mathematical Institute, Faculty of Mathematics and Physics, Charles University, \\ Sokolovská 83, 18675 Prague 8, Czech Republic \\ 2 Institute of Thermomechanics of the CAS, v. v. i., \\ Dolejškova 1402/5, 18200 Prague 8, Czech Republic \\ ${ }^{3}$ CTU Prague, Faculty of Civil Engineering, Thákurova 7, 16629 Prague 6, Czech Republic
}

\begin{abstract}
The Sedláček's turbine (known as SETUR) is an interesting hydraulic device that converts hydraulic energy into mechanical motion. The rotor in this turbine is blade-less and has a completely smooth shape. To find a physical explanation of its operation is an open problem, which we tackle here. Our theory is based on the idea that the fluid force on the rotor exists as a positive feedback to the orbital motion of the rotor. This is explained in a theoretical computation and supported by a numerical experiment.
\end{abstract}

Keywords: turbine, simulation.

\section{Introduction}

Rolling fluid turbine - also called SETUR - is a low-head water turbine invented by a Czech engineer Miroslav Sedláček in 1998 [1], [3]. Like most other turbines, it converts hydraulic power into rotary motion. It can be used to generate electricity or to directly induce motion in other hydraulic machines, e.g. cleaning brushes.

SETUR consist of a conical channel and a rotor with a shaft that connects it to stator e.g. by a ball joint. In the working mode, water flows through the channel and the fluid forces make the rotor to roll along the inner stator wall. The rotor hence performs (much like an inclined spinning top) both rotary and precession movement - the latter motion is used as a source of mechanical power and can be converted to electricity by a suitable generator. Interestingly, the rotor has completely smooth rotationally symmetric shape (commonly used are cones and hemispheres) and does not have any blades or buckets. The incoming flow may have even no prior angular momentum and in such case the direction of motion can be thought as random, or, more precisely, as depending merely on small symmetry flaws or initial perturbations. The hydraulic principle that powers SETUR is still unknown and is a subject of ongoing scientific research currently lagging behind experiments and applications. Simply put, it is known that SETUR is a functional design, but little is known about why it works. Naturally, this is a major obstacle in attempts to perfect this turbine.

The hydraulic mechanism can possibly be rather complicated and nonlinear [8]. However, we suggest a relatively simple novel explanation based on thesis [5]. While it might not be the definitive answer to this (perhaps much more difficult) problem, it leads to analytically tractable results and agrees with both numerical simulations and experimental observations.

\section{Theoretical analysis}

While being attached to the central symmetry axis, the rotor touches the conical channel at point $\boldsymbol{G}$ (see figure 1a) and this makes it slightly tilted (at angle about $5^{\circ}$ ). Alternatively, the rotor can be suspended from bellow by a cog which orbits the stator at a fixed distance $d$, making it parallel with the channel. This alternative design is perhaps less practical in terms of construction, but nonetheless it will be the case considered here, since it leads to simpler mathematics.

Let us call the angular speed of precession $\Omega$ and the angular speed of rotation $\Omega_{\text {rot }}$. We assume that the rotor maintains no-slip contact at $\boldsymbol{G}$ with the stator, which gives the relation

$$
\Omega_{\mathrm{rot}} R_{\mathrm{rot}}=\Omega d
$$




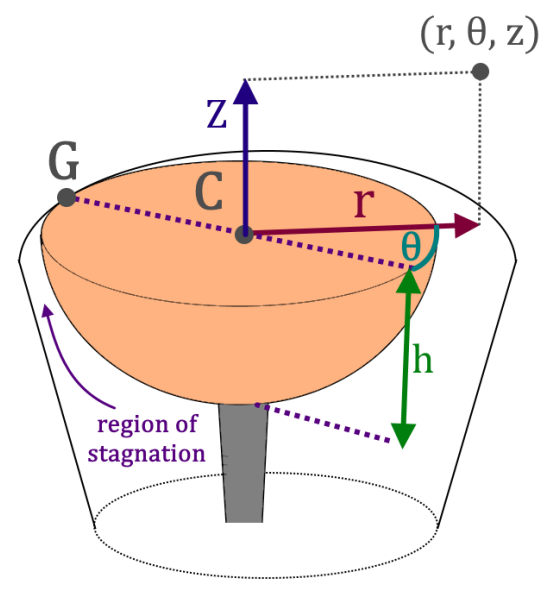

(a) SETUR viewed from a side.
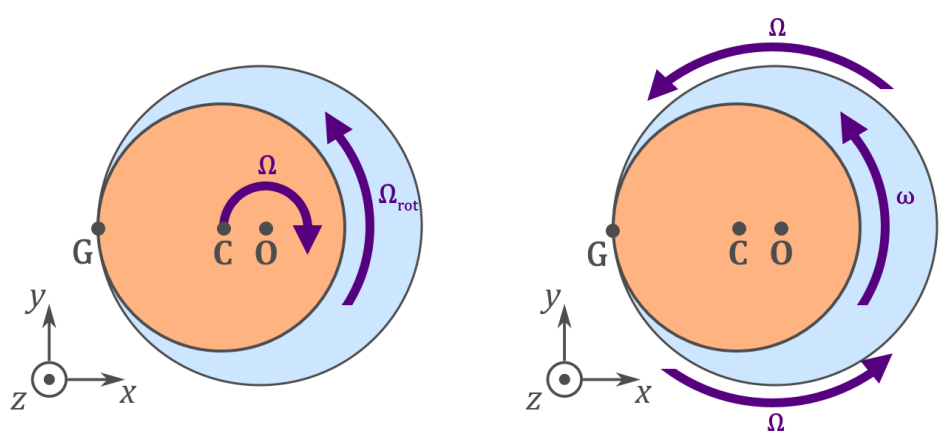

(b) SETUR (viewed from the top) with arrows depicting the movement in the laboratory frame (left) and the co-precessing frame (right).

where $R_{\text {rot }}, R_{\text {stat }}$ are the radii of the rotor and stator, respectively, and $d=R_{\text {stat }}-R_{\text {rot }}$ is the precession orbital radius. To better analyze the working mode of SETUR, it is helpful to change coordinates by switching into the co-precessing frame of reference. This is the frame which revolves in such a way as to follow the preceding motion of the rotor. More explicitly, it can be imagined in figure 1a as being an observer standing at $\boldsymbol{O}$ and looking towards $\boldsymbol{C}$ at all times. In this new frame, the fluid domain appears fixed in time, which is a great asset in theoretical discussions and in numerical simulations as well. Assuming that coordinate axes and orientation of $\Omega$ are chosen as in figure $1 \mathrm{~b}$, the transformation from the co-pressing frame (unprimed) to the laboratory frame (primed) is given by

$$
\boldsymbol{x}^{\prime}=\left[\begin{array}{ccc}
\cos \Omega t & \sin \Omega t & 0 \\
-\sin \Omega t & \cos \Omega t & 0 \\
0 & 0 & 1
\end{array}\right] \boldsymbol{x} .
$$

More important is the transformation of velocities

$$
\boldsymbol{v}^{\prime}=\boldsymbol{v}-\Omega \hat{z} \times \boldsymbol{x} .
$$

Inside the co-pressing frame, the stator and rotor will both appear to revolve around their axes in the same direction and with rates $\Omega$ and $\omega$, respectively, with

$$
\Omega R_{\text {stat }}=\omega R_{\text {rot }}
$$

due to the no-slip condition. Note that this equation relies on the assumption that the rotor be in a rotating steady state, which means that the turbine is in a working mode. Another important difference between the two frames of reference is that the incoming fluid has non-zero angular momentum in the co-precessing frame, being subjected to the relative solid body vortex of angular speed $\Omega$ corresponding to zero angular momentum in the laboratory frame.

Let us now analyze the stationary working mode, assuming that $\Omega$ is constant. We would like to analyze the flow field using stationary incompressible Navier-Stokes equations in the co-precessing 
frame. (Note that in the laboratory frame, the geometry is changing periodically and, therefore, the assumption of stationarity would not be meaningful.) The system of partial differential equations can be written succinctly as follows [6]:

$$
\begin{aligned}
& (\boldsymbol{v} \cdot \nabla) \boldsymbol{v}=-\nabla P+\boldsymbol{f}_{\mathrm{Cor}}+\nu \Delta \boldsymbol{v}, \\
& \operatorname{div} \boldsymbol{v}=0 .
\end{aligned}
$$

Here, $\boldsymbol{v}$ is the Eulerian velocity field, $\boldsymbol{f}_{\text {Cor }}$ is the Coriolis force

$$
\boldsymbol{f}_{\mathrm{Cor}}=2 \Omega \hat{z} \times \boldsymbol{v}
$$

and finally $P$ is the potential

$$
P=\frac{p}{\varrho}-\frac{1}{2} \Omega^{2} \xi^{2}+g z
$$

where $p[\mathrm{~Pa}]$ is the hydro-dynamical pressure, $\varrho \doteq 10^{3} \mathrm{~kg} / \mathrm{m}^{3}$ is density of water, $\xi[\mathrm{m}]$ is the distance from the central symmetry axis, $g \doteq 9.81 \mathrm{~m} / \mathrm{s}^{2}$ is the gravitational constant and $\nu \doteq 10^{-6} \mathrm{~m}^{2} / \mathrm{s}$ is the kinematic viscosity of water. Potential $P$ takes into account the pressure in the fluid, the centrifugal force and gravity, which are all conservative forces. Let us, for simplicity, assume freeslip boundary condition of the fluid at the rotor and stator walls, which is plausible outside of boundary layers [10].

Before proceeding further, let us set the characteristic speed $v_{\text {char }}$ of the flow as

$$
v_{\text {char }}=\frac{Q}{\rho A},
$$

where $A=\pi\left(R_{\text {stat }}^{2}-R_{\text {rot }}^{2}\right)$ is the inflow area and $Q[\mathrm{~kg} / \mathrm{s}]$ is the discharge. It will be useful for us to consider the following three dimensionless variables:

$$
\hat{d}=\frac{d}{R_{\text {rot }}}, \quad \hat{\Omega}=\frac{R_{\text {rot }} \Omega}{v_{\text {char }}}, \quad \operatorname{Re}=\frac{v_{\text {char }} d}{\nu},
$$

where Re stands for the Reynolds number.

In what follows, we would like to find the $y$-component of the net fluid force $\boldsymbol{F}$ acting upon the rotor. (Due to the assumption of stationarity, the $y$-component plays the role of the force in direction of precession.) To this end, let $r, \theta, z$ be the cylindrical coordinates measured from the rotor symmetry axis, as depicted in figure 1a. In these coordinates the rotor surface can be parameterized as

$$
\Gamma_{\text {rot }}=\left\{r=r_{0}(z) ; \quad z \in[-h, 0]\right\},
$$

with the surface normal given by

$$
\boldsymbol{n}=\left[\begin{array}{c}
\cos \gamma \cos \theta \\
\cos \gamma \sin \theta \\
-\sin \gamma
\end{array}\right]
$$

The coefficient

$$
\gamma=\arctan \left(\frac{\mathrm{d} r_{0}}{\mathrm{~d} z}\right)
$$

expresses the inclination of surface element at $r_{0}(z)$ with respect to $x y$-plane. We then have

$$
\begin{aligned}
F_{y} & =-\int_{\Gamma_{\text {rot }}} \rho P n_{y} \mathrm{~d} S \\
& =-\left.\int_{-h}^{0} \int_{-\pi}^{\pi} \rho P\right|_{r=r_{0}} n_{y} \frac{r_{0}}{\cos \gamma} \mathrm{d} \theta \mathrm{d} z \\
& =-\left.\rho \int_{-h}^{0} r_{0} \int_{-\pi}^{\pi} P\right|_{r=r_{0}} \sin \theta \mathrm{d} \theta \mathrm{d} z .
\end{aligned}
$$


Note that only the $p$-dependent part of the potential plays a role, since the other parts are integrated to zero. Translating this result into the inertial frame, where the rotor orbits the central symmetry axis with speed $\Omega d$, the power $\dot{W}$ exerted on the rotor becomes

$$
\dot{W}=F_{y} \Omega d
$$

We thus see that the power is directly related to the sine mode of pressure. More precisely, when the sine mode is positive, the fluid exerts a stopping force on the rotor, which would have to be compensated (e.g. by an external engine), for the rotor would decelerate otherwise. On the other hand, when the sine mode is negative, the fluid pushes the rotor onwards and power has to be extracted (e.g. by a generator), for else the rotor would accelerate until a new balance compensated by dissipation is reached.

We are now prepared to propose a theoretical explanation of the hydraulic mechanism behind the rolling turbine. Our first observation is that, due to the rotor shape (see figure 1a), the channel between the stator and the rotor is narrowest in the cross-section of contact, at $z=0$. The channel then expands, causing the fluid to decelerate. Due to the off-centered position of the rotor, the expansion is not uniform and it is most significant near $\theta= \pm \pi$. In this region $v_{z}$ becomes smaller in magnitude (i.e. "less negative") than in the wider part of the channel. We may call it a region of stagnation (this will be verified numerically in the upcoming section). This variation in velocity then naturally also leads to a variation in pressure, but, since the fluid is rotating in this frame, the variation becomes shifted by $90^{\circ}$, yielding $F_{y}>0$ in equation (13).

To elaborate this connection, let us write

$$
v_{\theta}=u_{\theta}+r \Omega
$$

We will now make a simplifying assumption that $v_{z}, v_{r}$ are even functions with respect to $\theta$ while $u_{\theta}$ being odd. To motivate this, consider an error

$$
\epsilon=\frac{\left\|v_{r, \text { odd }}\right\|+\left\|u_{\theta, \text { even }}\right\|+\left\|v_{z, \text { odd }}\right\|}{v_{\text {char }}},
$$

where $\|\cdot\|$ denotes some functional norm (e.g. a 1,2 Sobolev norm) and

$$
f_{\text {even }}(r, \theta, z)=\frac{f(r, \theta, z)+f(r,-\theta, z)}{2}, \quad f_{\text {odd }}(r, \theta, z)=\frac{f(r, \theta, z)-f(r,-\theta, z)}{2}
$$

represent the even and odd parts of an arbitrary function $f$ w.r.t. $\theta$. For $\hat{\Omega}=0$ the domain is symmetric with respect to reflection in the $z x$-plane and thus $v_{\theta}=0$ and $v_{r}$ and $v_{z}$ are symmetric with respect to that reflection, i.e. $\left.\epsilon\right|_{\hat{\Omega}=0}=0$. For a moment, let us consider $d$, the rotor offset, to be a free parameter in the range $\left[0, R_{\text {stat }}-R_{\text {rot }}\right]$ and consider angular speed of inflow vortex $\Omega$ as an independent variable. Since the preceding argument holds true for any $d$, we have, moreover, that $\left.\frac{\partial \epsilon}{\partial d}\right|_{\hat{\Omega}=0}=0$. On the other hand, setting $\hat{d}=0$ while $\hat{\Omega} \neq 0$, i.e. aligning the rotor with the central symmetry axis, makes the domain rotationally symmetric and thus $v_{\theta}=r \Omega$ and $v_{r}$, $v_{z}$ become independent of $\theta$. This leads to $\left.\epsilon\right|_{\hat{d}=0}=0$, and since this holds for any small enough rotation rates $\hat{\Omega}$, we obtain, moreover, that $\left.\frac{\partial \epsilon}{\partial \hat{\Omega}}\right|_{d=0}=0$. Altogether, we have that

$$
\begin{aligned}
\epsilon(\hat{\Omega}, \hat{d}) & =\epsilon(0,0)+\left.\frac{\partial \epsilon}{\partial \hat{\Omega}}\right|_{\hat{\Omega}=\hat{d}=0} \hat{\Omega}+\left.\frac{\partial \epsilon}{\partial \hat{d}}\right|_{\hat{\Omega}=\hat{d}=0} \hat{d}+\mathcal{O}\left(\hat{\Omega}^{2}+\hat{d}^{2}\right) \\
& =0+0+0+\mathcal{O}\left(\hat{\Omega}^{2}+\hat{d}^{2}\right) \\
& =\mathcal{O}\left(\hat{\Omega}^{2}+\hat{d}^{2}\right) .
\end{aligned}
$$

Setting $\epsilon=0$ can be then understood as a first order approximation when the two borderline symmetric cases are sufficiently "close" (i.e. for $\hat{\Omega}, \hat{d} \ll 1$ ), which is where the assumption that $v_{z}$ and $v_{r}$ be even functions of $\theta$ while $u_{\theta}$ an odd function should be satisfied. 
Expressing the $z$-component of (5), we find

$$
\Omega \frac{\partial v_{z}}{\partial \theta}+\left(v_{r} \frac{\partial v_{z}}{\partial r}+\frac{u_{\theta}}{r} \frac{\partial v_{z}}{\partial \theta}+v_{z} \frac{\partial v_{z}}{\partial r}-\nu \Delta v_{z}\right)=-\frac{\partial P}{\partial z} .
$$

Now, let us multiply by $\sin \theta$ and integrate from $-\pi$ to $\pi$, noting that the whole expression in the brackets is even with respect to $\theta$ and thus disappears. We get

$$
\Omega \int_{-\pi}^{\pi} \frac{\partial v_{z}}{\partial \theta} \sin \theta \mathrm{d} \theta=-\int_{-\pi}^{\pi} \frac{\partial P}{\partial z} \sin \theta \mathrm{d} \theta
$$

and, after integration by parts,

$$
\Omega \int_{-\pi}^{\pi} v_{z} \cos \theta \mathrm{d} \theta=\int_{-\pi}^{\pi} \frac{\partial P}{\partial z} \sin \theta \mathrm{d} \theta .
$$

An analogous computation for the radial component yields

$$
\Omega \int_{-\pi}^{\pi} v_{r} \cos \theta \mathrm{d} \theta=\int_{-\pi}^{\pi} \frac{\partial P}{\partial r} \sin \theta \mathrm{d} \theta
$$

Next, we multiply (22) by $\tan \gamma$ and sum together with (21). Evaluating at $r=r_{0}(z)$ and using the non-penetration boundary, $v_{z} \tan \gamma=v_{r}$ at the rotor surface, we obtain that

$$
\begin{aligned}
& \left.\frac{\Omega}{\cos ^{2} \gamma} \int_{-\pi}^{\pi} v_{z}\right|_{r=r_{0}(z)} \cos \theta \mathrm{d} \theta \\
& =\int_{-\pi}^{\pi}\left(\frac{\partial P}{\partial z}+\tan \gamma \frac{\partial P}{\partial r}\right)_{r=r_{0}(z)} \sin \theta \mathrm{d} \theta \\
& =\left.\frac{\mathrm{d}}{\mathrm{d} z} \int_{-\pi}^{\pi} P\right|_{r=r_{0}(z)} \sin \theta \mathrm{d} \theta .
\end{aligned}
$$

Naturally, we would like to use this partial result to calculate the power. To do this, let us first define dimensionless variables

$$
\begin{aligned}
C_{k}(z) & :=\left.\frac{\rho A}{Q} \int_{-\pi}^{\pi} v_{z}\right|_{r=r_{0}} \cos k \theta \mathrm{d} \theta, \quad k \in \mathbb{N}_{0} \\
L(z) & :=\frac{h d}{A^{2}} \int_{z}^{0} r_{0} \mathrm{~d} z .
\end{aligned}
$$

Integration by parts in (13) leads to

$$
\begin{aligned}
F_{y} & =\left.\frac{\rho A^{2}}{h d} \int_{-h}^{0} \frac{\mathrm{d} L}{\mathrm{~d} z} \int_{-\pi}^{\pi} P\right|_{r=r_{0}} \sin \theta \mathrm{d} \theta \\
& =-\left.\frac{\rho A^{2}}{h d} \int_{-h}^{0} L \frac{\mathrm{d}}{\mathrm{d} z} \int_{-\pi}^{\pi} P\right|_{r=r_{0}} \sin \theta \mathrm{d} \theta
\end{aligned}
$$

where boundary terms disappear, since $L(0)=0$ and, on the other hand

$$
\left(\left.\int_{-\pi}^{\pi} P\right|_{r=r_{0}} \sin \theta\right)_{z=-h}=0
$$

provided that $P$ is continuous and $r(-h)=0$. The latter assumption is valid for instance for a hemispherical rotor. If we wanted to be more precise, we would say $r(-h)=r_{\text {shaft }}$, where $r_{\text {shaft }}$ is the radius of the shaft that support the rotor, see Fig. 1a. However, the shaft can be very thin and is not relevant for the working mechanism that we are trying to illustrate. Finally, Eqs. (23, 24) lead to the expression for the force

$$
F_{y}=-\frac{A Q \Omega}{h d} \int_{-h}^{0} \frac{L C_{1}}{\cos ^{2} \gamma} \mathrm{d} z
$$


and power, using Eq. (14),

$$
\dot{W}=-A Q \Omega^{2} \int_{-h}^{0} \frac{L C_{1}}{\cos ^{2} \gamma} \frac{\mathrm{d} z}{h} .
$$

This demonstrates that there is, indeed, a direct link between the power and the first cosine mode of $v_{z}$. More specifically, if our assumption about the region of stagnation holds, then $C_{1}<0$ and hence the right hand side in (28) is positive, giving positive feedback to the precession driving the turbine. This can be seen as an instability leading to a stable non-equilibrium stationary state (the working mode). From the perspective of thermodynamics, it should be emphasized that such behavior is possible only because the system is open, owing to the presence of constant discharge $Q>0$. In summary, from the assumption about the region of stagnation and the assumption about symmetries of the velocity field it follows that positive feedback to the rotor motion develops, which drives the precession of the rotor and yields positive power.

This whole computation was done for $\hat{\Omega} \ll 1$ and represents only the least order (quadratic) non-trivial approximation of power w.r.t. $\Omega$. It can be seen from the numerical analysis and experimental observations (see below) that the next, cubic, term in the expansion of $\dot{W}$ in $\Omega$ acts against the motion of the rotor. This corresponds to an additional force term akin to Newton's drag formula

$$
F_{y}=-\frac{A Q \Omega}{h d} \int_{-h}^{0} \frac{L C_{1}}{\cos ^{2} \gamma} \mathrm{d} z-\zeta \Omega^{2} \operatorname{sgn} \Omega
$$

where $\zeta>0$ plays a role of a friction coefficient. Addition of this force serves as a remedy for the assumption of free-slip boundary conditions made above. An improved expression for power then becomes

$$
\dot{W}=c\left(1-\frac{|\Omega|}{\Omega_{\mathrm{f}}}\right) A Q \Omega^{2}
$$

where

$$
c=-\int_{-h}^{0} \frac{L C_{1}}{\cos ^{2} \gamma} \frac{\mathrm{d} z}{h}, \quad \Omega_{\mathrm{f}}=\frac{c A Q}{\zeta d}
$$

are two dimensionless parameters. These are determined by construction of the turbine and by parameters of the entering flow field. In this model, $\Omega_{\mathrm{f}}$ is the precession speed of a "free" working mode - the state where the resistance from generator is zero and no power is produced. Friction forces act against the motion of the rotor and a Newtonian drag formula improves agreement with experimental data even for higher rotation rates. As so far, this analysis assumed zero angular momentum of the incoming flow in the inertial frame. The simplest way how to generalize equation (30) to a non-zero incoming solid body vortex with angular velocity $-\vartheta \hat{\boldsymbol{z}}$ is by substituting $\Omega \mapsto \Omega-\vartheta$ in $(27)$ and (29), which leads to

$$
\dot{W}=c\left(1-\frac{|\Omega-\vartheta|}{\Omega_{\mathrm{f}}}\right) A Q \Omega(\Omega-\vartheta) .
$$

This model then predicts that SETUR can operate with precession going either in or against the direction of incoming vortex. It would then seem from (32) that power decreases when the signs of $\Omega$ and $\vartheta$ coincide, but the opposite is true in the sense that the maximal attainable power is greater.

\section{Simulation}

In order to make a $3 \mathrm{~d}$ simulation of the turbine, we employed finite-element method in FEniCS. Following the same strategy used in Sec. 2, first, we prescribed a constant speed of precession and then switched into the co-precessing frame, where the geometry appears static. This allows for computations with a fixed mesh, which greatly simplifies the problem. As for our physical model, we have chosen the incompressible time-dependent Navier-Stokes equations with the Coriolis term:

$$
\begin{aligned}
& \frac{\partial \boldsymbol{v}}{\partial t}+(\boldsymbol{v} \cdot \nabla) \boldsymbol{v}=-\nabla P+\boldsymbol{f}_{\mathrm{Cor}}+\nu \Delta \boldsymbol{v}, \\
& \operatorname{div} \boldsymbol{v}=0 .
\end{aligned}
$$


Table 1: Results of numerical simulations.

\begin{tabular}{|c|c|c|c|c|}
\hline$f[\mathrm{~Hz}]$ & $\Omega[\mathrm{rad} / \mathrm{s}]$ & $\hat{\Omega}$ & $\dot{W}[\mathrm{~W}]$ & $c$ \\
\hline \hline 0.02 & 0.126 & 0.129 & $2.2 \mathrm{e}-5$ & $5.33 \mathrm{e}-2$ \\
\hline 0.2 & 1.257 & 1.283 & $2.3 \mathrm{e}-3$ & $5.56 \mathrm{e}-2$ \\
\hline 0.5 & 3.142 & 3.208 & $1.4 \mathrm{e}-2$ & $5.43 \mathrm{e}-2$ \\
\hline 1.0 & 6.283 & 6.415 & $4.3 \mathrm{e}-2$ & $4.16 \mathrm{e}-2$ \\
\hline
\end{tabular}

For the rotor we have chosen a truncated conical shape with biggest radius $R_{\text {rot }}=0.125 \mathrm{~m}$ while for the stator a cone with biggest radius $R_{\text {rot }}=0.135 \mathrm{~m}$. The Reynolds number, defined in (9), was 1224. Although the geometry is relatively simple, it has a problematic property: the presence of a contact point between the rotor and stator, which makes mesh generation difficult. Therefore, we decided to crop the geometry by a transversal plane in order to exclude that point. Consequently, the inflow area is an annular region between two circles almost in touch. Of course the mesh needed to be refined near the narrowed region. As for the discretization, we employed Taylor-hood elements and implicit Euler time-stepping. We prescribed no-slip boundary condition at the walls of rotor and stator

Dirichlet inflow at the top

$$
\left.\boldsymbol{v}\right|_{\Gamma_{\text {rot }} \cup \Gamma_{\text {stat }}}=0
$$

$$
\left.\boldsymbol{v}\right|_{\Gamma_{\mathrm{in}}}=-\frac{Q}{\rho A} \hat{\boldsymbol{z}}+\boldsymbol{\Omega} \times \boldsymbol{x},
$$

and a boundary condition of do-nothing type at the bottom [2].

$$
\left.\mathbb{T} \boldsymbol{n}\right|_{\Gamma_{\text {out }}}=0
$$

(where $\mathbb{T}=-\nabla p \mathbb{I}+\mu\left(\nabla \boldsymbol{v}+\nabla \boldsymbol{v}^{T}\right)$ is the Cauchy stress tensor and $\boldsymbol{n}$ is the outward surface normal. The output of the simulation was to measure the power on the rotor using formula (14). (Note that we assume that the turbine be in a steady working state. Readers interested in the onset of the instability leading to that steady state can consult work [1].)

This numerical experiment confirmed the existence of the positive-feedback mechanism and also validity of the square law

$$
\dot{W} \propto \hat{\Omega}^{2}
$$

for precession frequency $f$ in range $0.02 \mathrm{~Hz}-1.0 \mathrm{~Hz}$. Results of four simulations with different values of $\hat{\Omega}$ are listed in table 1 and displayed in figure 2. In all these simulations the power stabilized itself after some transition period and this value was used. In all cases, the power was positive, indicating that the positive feedback was stronger than friction losses. Table 1 also lists dimensionless parameter $c$ from the equation

$$
c=\frac{\dot{W}}{A Q \Omega^{2}} .
$$

The numerical simulations support results of the above analytical discussion and the underlying assumptions.

We realize, however, that the presented simulation has a certain weak spot, namely that the velocities at the inflow had to be prescribed. It could be that in reality the incoming flow is much faster in the narrow region of the cross-section so that the effect of uneven expansion would cancel out. However, we believe that this is not case, since viscous forces prevent high velocities near the contact point. This argument seems to be somewhat analogical to the Joukowski condition for airfoils, where viscous forces are taken into account at the sharp trailing edge [6]. We plan to further asses this question in the future by making a numerical simulation in an extended domain (which poses a numerical challenge of dealing with a non-Lipschitz geometry at the contact point). 

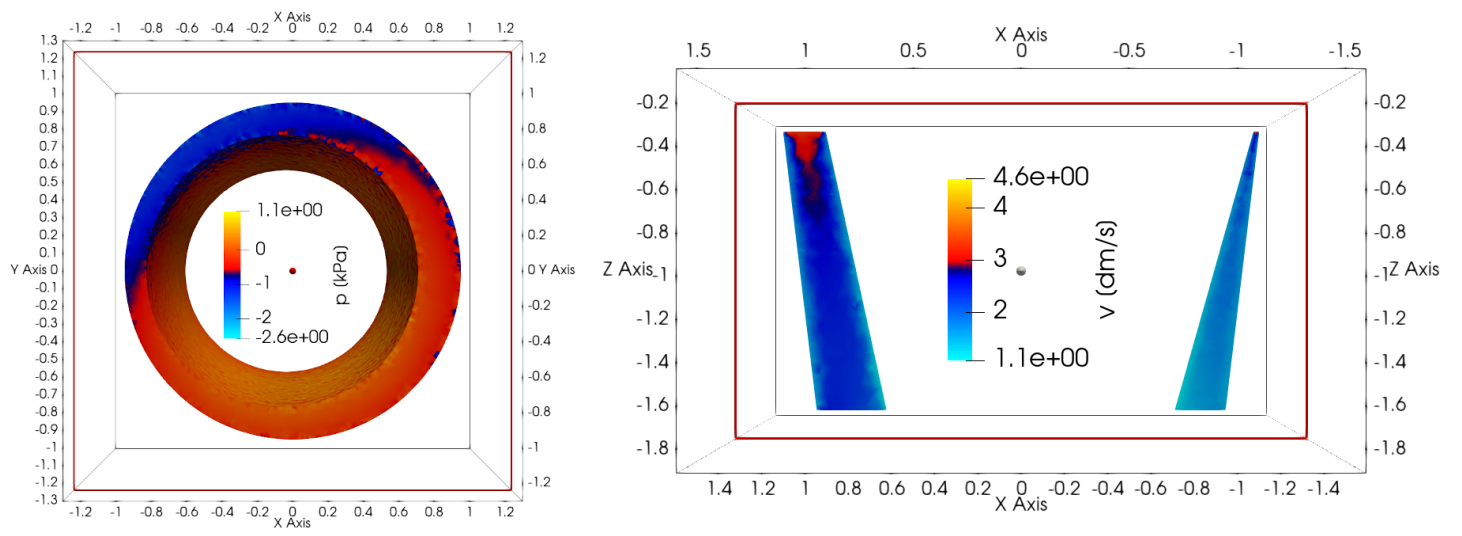

Figure 2: Left: Pressure field (top view). Region of decreased pressure in front of rotor (blue) can be clearly recognized. Right: Magnitude of velocity (side view) - although the inlet velocity was the same at the top, geometrical expansion near the gap (right) is much greater, causing the fluid to decelerate. Stagnation on the right is clearly visible. Output of the simulation for $f=0.2 \mathrm{~Hz}$. (created using Paraview [4])

\section{Conclusion}

The rolling fluid turbine is a bladeless hydraulic device capable to efficiently convert small hydraulic heads into electrical power. In this article, we have proposed a novel theory capable to explain the working mode of the turbine by analytical means. Our theory suggests that the work exerted on the rotor comes from a combination of the vortex motion in the co-precessing frame (or periodic temporal oscillations in the laboratory frame) and unevenness of the $z$-component of the velocity field. None of these effects would generate significant variations in the pressure field by itself, but together they do. This occurs due to the quadratic nonlinearity in the convective term. Finally, this results in positive feedback on the rotor, which explains the peculiar behavior of SETUR in the quiescent state. We of course rely on several simplifying assumptions, but numerical simulations have been carried out supporting those assumptions. We believe that our theory explains how the rolling fluid turbine (SETUR) actually works.

In future we would like to address shape optimization of the rotor using our analytical results (28) and carry out simulation corresponding to higher values of power outputs. We would also like to investigate exergy losses in the turbine [9] and how the turbine reflects the principle of minimal entropy production [7] while approaching the steady rotating state.

\section{Acknowledgment}

We are grateful to Jaroslav Hron for his valuable comments on the diploma thesis on which this manuscript was based. O.K. and M.P. were supported by the Czech Grant Agency, project number 20-22092S. M.P. was supported by Charles University Research program No. UNCE/SCI/023.

\section{References}

[1] Beran, V., Sedláček, M. \& Maršík, F.: A new bladeless hydraulic turbine. Applied Energy. (2013). pp. 987-983.

[2] Braack, M. \& Mucha, P. B.: Directional do-nothing condition for the navier-stokes equations. Journal of Computational Mathematics. vol. 32 no. 5 (2014).

[3] Hostin, S., Sedláček, M. \& Špidla, J.: Small hydroelectric power plant, final report. Bratislava (1997). 
[4] James, A., Berk, G. \& Charles, L.: ParaView: An End-User Tool for Large Data Visualization. Elsevier (2005).

[5] Kincl, O.: Thermodynamic modeling of rolling fluid turbine. Master's thesis: Charles University: Prague (2020).

[6] Landau, L. \& Lifshitz, E. M.: Fluid Mechanics. Pergamon Press: Oxford (1987).

[7] Lebon, G., Jou, D. \& Vázquez, J. C.: Understanding Non-Equilibrium Thermodynamics: Foundations, Applications, Frontiers. SpringerLink: Springer e-Books. Springer London, Limited (2008). ISBN 9783540742524.

[8] Maršík, F., Falta, J. \& Sedláček, M.: Theoretical analysis and experimental verification of the rolling turbines performance. 7th International Conference on Heat Transfer, Fluid Mechanics and Thermodynamics: Anatolia, Turkey (2010).

[9] Pavelka, M., Klika, V., Vágner, P. \& Maršík, F.: Generalization of exergy analysis. Applied Energy. vol. 137 (2015). pp. 158 - 172. ISSN 0306-2619. doi: http://dx.doi.org/10.1016/j.apenergy.2014.09.071.

[10] Sommerfeld, A.: Mechanics of Deformable Bodies. Academic Press: (1997). 